\title{
ISSEC: inferring contacts among protein secondary structure elements using deep object detection
}

\author{
Qi Zhang ${ }^{1,2+}$, Jianwei Zhu ${ }^{1,2 \dagger}$, Fusong Ju ${ }^{1,2}$, Lupeng Kong ${ }^{1,2}$, Shiwei Sun ${ }^{1,2}$, Wei-Mou Zheng ${ }^{3}$ \\ and Dongbo $\mathrm{Bu}^{1,2^{*}}$ (])
}

\author{
*Correspondence: \\ dbu@ict.ac.cn \\ ${ }^{\dagger}$ Qi Zhang and Jianwei Zhu \\ have contributed equally. \\ ${ }^{1}$ Key Lab of Intelligent \\ Information Processing, Big \\ Data Academy, Institute \\ of Computing Technology, \\ Chinese Academy \\ of Sciences, Beijing 100190, \\ China \\ Full list of author information \\ is available at the end of the \\ article
}

\begin{abstract}
Background: The formation of contacts among protein secondary structure elements (SSEs) is an important step in protein folding as it determines topology of protein tertiary structure; hence, inferring inter-SSE contacts is crucial to protein structure prediction. One of the existing strategies infers inter-SSE contacts directly from the predicted possibilities of inter-residue contacts without any preprocessing, and thus suffers from the excessive noises existing in the predicted inter-residue contacts. Another strategy defines SSEs based on protein secondary structure prediction first, and then judges whether each candidate SSE pair could form contact or not. However, it is difficult to accurately determine boundary of SSES due to the errors in secondary structure prediction. The incorrectly-deduced SSEs definitely hinder subsequent prediction of the contacts among them.
\end{abstract}

Results: We here report an accurate approach to infer the inter-SSE contacts (thus called as ISSEC) using the deep object detection technique. The design of ISSEC is based on the observation that, in the inter-residue contact map, the contacting SSEs usually form rectangle regions with characteristic patterns. Therefore, ISSEC infers interSSE contacts through detecting such rectangle regions. Unlike the existing approach directly using the predicted probabilities of inter-residue contact, ISSEC applies the deep convolution technique to extract high-level features from the inter-residue contacts. More importantly, ISSEC does not rely on the pre-defined SSEs. Instead, ISSEC enumerates multiple candidate rectangle regions in the predicted inter-residue contact map, and for each region, ISSEC calculates a confidence score to measure whether it has characteristic patterns or not. ISSEC employs greedy strategy to select nonoverlapping regions with high confidence score, and finally infers inter-SSE contacts according to these regions.

Conclusions: Comprehensive experimental results suggested that ISSEC outperformed the state-of-the-art approaches in predicting inter-SSE contacts. We further demonstrated the successful applications of ISSEC to improve prediction of both interresidue contacts and tertiary structure as well.

Keywords: Protein structure, Secondary structure elements, Inter-SSE contacts author(s) and the source, provide a link to the Creative Commons licence, and indicate if changes were made. The images or other third party material in this article are included in the article's Creative Commons licence, unless indicated otherwise in a credit line to the material. If material is not included in the article's Creative Commons licence and your intended use is not permitted by statutory regulation or exceeds the permitted use, you will need to obtain permission directly from the copyright holder. To view a copy of this licence, visit http:// creativecommons.org/licenses/by/4.0/. The Creative Commons Public Domain Dedication waiver (http://creativecommons.org/publi cdomain/zero/1.0/) applies to the data made available in this article, unless otherwise stated in a credit line to the data. 


\section{Background}

Proteins play important roles in a large variety of biological processes. The biological roles of proteins are mainly determined by their three dimensional structures (called tertiary structures), making resolving protein structures highly desirable. The experimental technologies to resolve protein tertiary structures, such as X-ray crystallography, NMR spectroscopy, and cryo-electron microscopy, have achieved great successes; however, these technologies are usually time-consuming. Thus, predicting protein structure from amino acid sequence is of great importance $[1,2]$.

Protein structures are stabilized by both local and global interactions among composing residues; thus, understanding of contacts among residues could effectively facilitate prediction of the correct protein fold [3,4]. Some recent studies have shown significant improvement in the prediction of inter-residue contacts [5]; however, the application of the predicted residue contact to build protein structure is still far from satisfactory $[3,6$, 7]. The potential reasons lie at the errors in the predicted residue-level contacts and the limitations in exploitation of these contacts.

In this study, we focus on the prediction of inter-SSE contacts. The prediction of interSSE contacts is of great importance as inter-SSE contacts carry coarse-grain information of tertiary structure and thus could effectively facilitate protein structure prediction [810]. In addition, compared with inter-residue contacts, the inter-SSE contacts are much more robust, making it more reliable to predict.

A variety of methods have been developed for the prediction of inter-SSE contacts, which could be roughly divided into two categories, namely, prediction of $\alpha-\alpha$ contacts [11-15] and prediction of $\beta-\beta$ contacts [16-18]. For example, bbcontacts focuses on the prediction of $\beta-\beta$ parallel and antiparallel contacts [7]. It employs the hidden Markov model (HMM) technique to integrate signals covering the predicted inter-residue contacts and the predicted secondary structure. As it directly uses the predicted probability of inter-residue contacts, bbcontacts suffers from the excessive noises existing in these contacts. In contrast to bbcontacts, HHConPred aims to predict $\alpha-\alpha$ contacts [19]. It infers the contact between two helices via checking whether these helices show ridge pattern in the inter-residue contact maps. However, as this approach needs pre-defined SSEs, it suffers from the errors of predicted secondary structure. Besides, both of these two approaches were designed for a single type of inter-SSE contacts only and thus could not apply for the proteins with mixed types of inter-SSE contacts.

We here report an accurate approach (called ISSEC) to the prediction of inter-SSE contacts. ISSEC is rooted in the observation that, in the inter-residue contact map, the contacting SSEs usually form a rectangle region with characteristic patterns (Fig. 1 and Additional file 1: Fig. S1). For example, two contacting parallel $\beta$-strands often form a diagonal line, whereas two contacting anti-parallel $\beta$-strands form an anti-diagonal line. In contrast, two contacting helices usually form a dashed line.

ISSEC applies the object detection technique to detect the rectangle regions with characteristic patterns, and infers inter-SSE contacts based on these regions. ISSEC has the following advantages: (1) It uses deep convolution to extract high-level features from the predicted inter-residue contacts and thus could tolerate excessive noises in these contacts. (2) ISSEC examines multiple overlapping regions in the predicted contact map and thus could get rid of the dependency on the pre-defined SSEs. 


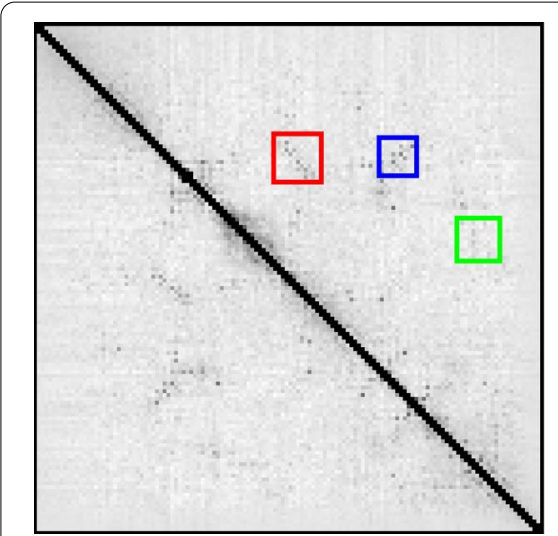

Patterns of inter-residue contacts

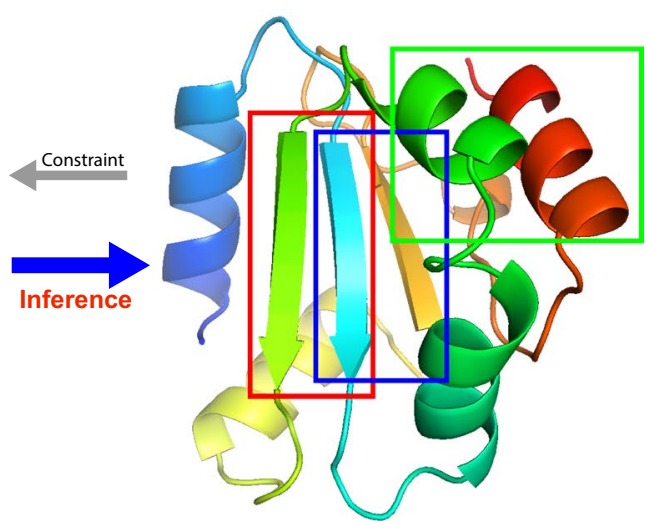

Inter-SSE contacts in 3D structure

Fig. 1 Characteristic patterns formed by contacting SSEs in inter-residue contact map. Two contacting parallel $\beta$-strands often form a diagonal line (rectangle in red), whereas two contacting anti-parallel $\beta$-strands form an anti-diagonal line (rectangle in blue). In contrast, two contacting helices usually form a dashed line (rectangle in green). These characteristic patterns could be used to infer inter-SSE contacts

(3) ISSEC could predict multiple types of inter-SSE contact simultaneously. Experimental results on PSICOV [20], CASP11 datasets, and membrane proteins suggest that ISSEC significantly outperformed the existing approaches in prediction accuracy. Furthermore, we successfully applied the predicted inter-SSE contacts to improve the prediction of inter-residue contacts and tertiary structure as well.

\section{Results}

We first explain the concept of ISSEC using protein $3 a 4 t A$ as a concrete example. Next we show the prediction accuracy of ISSEC on a variety of datasets, including PSICOV dataset, TEST1000 dataset, and transmembrane proteins. Finally, we show the application of ISSEC to improve inter-residue prediction and tertiary structure prediction as well.

\section{Datasets}

From the proteins in PDB25 (Released in February, 2015), we randomly selected 1,000 proteins to construct a test set called TEST1000. The other test sets include: (1) PSICOV118 dataset: consisting of 118 proteins (after excluding 32 proteins without inter-SSE contacts from PSICOV dataset). (2) Mem30 dataset: consisting of 30 transmembrane proteins obtained from MemConP [15]. (3) Mem11 dataset: consisting of 11 transmembrane proteins [19]. (4) BetaSheet186: consisting of 186 proteins that contains $\beta-\beta$ contacts [17].

The training set was also constructed based on PDB25. To guarantee low sequence identity between training and testing set, we excluded the proteins with sequence identify over $25 \%$ with any protein in the test sets and finally obtained a training set with 9241 proteins. 


\section{The concept of ISSEC using protein $3 a 4 t A$ as an example}

The protein $3 \mathrm{a} 4 \mathrm{tA}$ is an $\alpha-\beta$ protein that consists of 274 residues, forming $20 \alpha$ and $\beta$ SSEs, i.e., $\quad E_{1}-H_{1}-E_{2}-E_{3}-E_{4}-H_{2}-E_{5}-H_{3}-E_{6}-H_{4}-E_{7}-H_{5}-E_{8}-H_{6}-E_{9}-H_{7}-E_{10}-E_{11}-E_{12}-E_{13}$ (Fig. 2). Here $E$ represents a $\beta$ strand and $H$ represents an $\alpha$ helix. This protein has a total of 12 inter-SSE contacts, including $2 \alpha-\alpha$ contacts $\left(H_{2}-H_{3}\right.$ and $\left.H_{6}-H_{7}\right), 4 \beta-\beta$ parallel contacts $\left(E_{5}-E_{6}, E_{5}-E_{8}, E_{6}-E_{7}\right.$, and $\left.E_{8}-E_{9}\right)$, and $6 \beta-\beta$ anti-parallel contacts $\left(E_{1}-E_{3}\right.$, $E_{1}-E_{4}, E_{2}-E_{3}, E_{9}-E_{13}, E_{10}-E_{13}$ and $\left.E_{12}-E_{13}\right)$.

For this protein, ISSEC first predicted probabilities of inter-residue contacts (shown in lower-left triangle of Fig. 2a), which contain a great amount of noises when compared with the true residue contacts (upper-right triangle). Based on these predicted probabilities of inter-residue contacts, ISSEC identified 12 inter-SSE contacts, including 9 positive predictions (rectangle regions in red) and 3 false positives (rectangle regions in green). In addition, 3 inter-SSE contacts $\left(E_{1}-E_{3}, E_{2}-E_{3}\right.$ and $\left.H_{2}-H_{3}\right)$ were missed by ISSEC.

Besides the type of inter-SSE contacts, ISSEC also reports their positions along with confidence scores. Figure 2c shows an example: ISSEC identified an $\alpha-\alpha$ contact with confidence score of 0.80 . The position of the identified rectangle region matches perfectly with the true position of the contacting SSEs (shown in upper-right triangle). For this rectangle region, ISSEC also reports mask to show the contacting residues. However, the true contacting residues form a dashed line while ISSEC prefers to report a continuous line. This is the reason why ISSEC sets a relatively small weight for the mask loss.

\section{Accuracy of inter-SSE contact prediction}

We first evaluated ISSEC's performance on proteins that contain single type of interSSE contacts. Next, we tested ISSEC on proteins that contain multiple types of inter-SSE contacts.

\section{Prediction accuracy of $\alpha-\alpha$ contacts for membrane proteins}

Most membrane proteins are composed of only $\alpha$-helices. Thus, the accurate prediction of $\alpha-\alpha$ contacts should greatly facilitate the prediction of tertiary structure for membrane proteins [21].

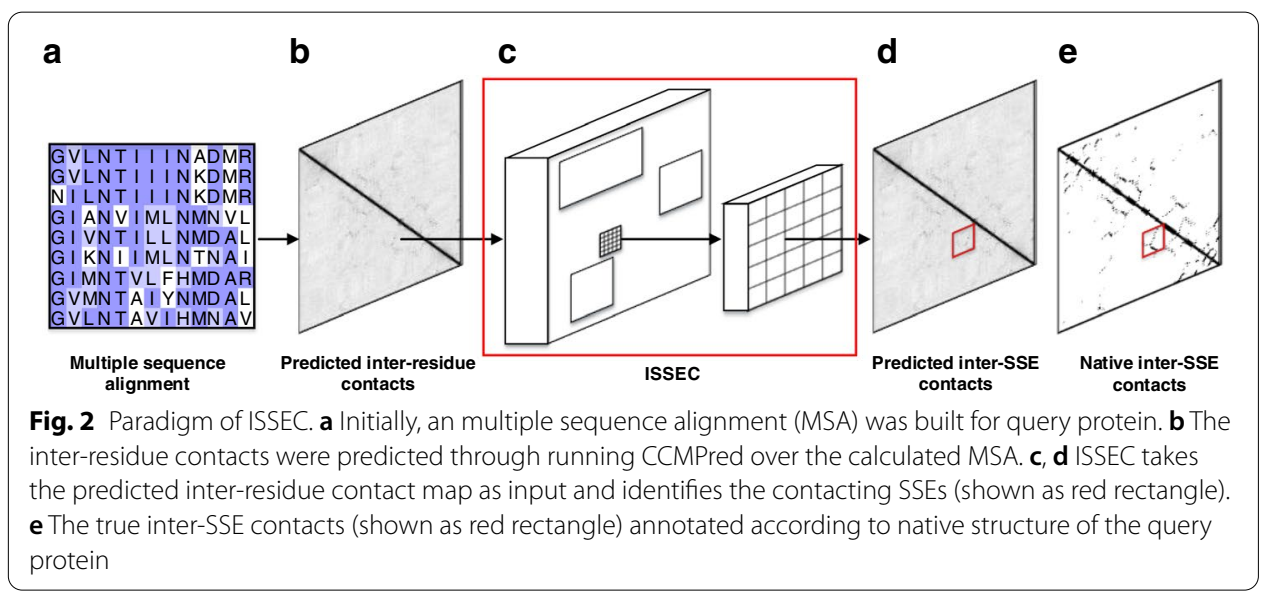




\begin{tabular}{|c|c|c|c|c|c|c|}
\hline \multirow[t]{2}{*}{ Method } & \multicolumn{3}{|l|}{ Mem30 } & \multicolumn{3}{|l|}{ Mem11 } \\
\hline & Precision (\%) & Recall (\%) & F-measure (\%) & Precision (\%) & Recall (\%) & F-measure (\%) \\
\hline TMhhep & 61.85 & 15.48 & 23.91 & 36.36 & 31.60 & 30.81 \\
\hline MemConP & 70.68 & 24.97 & 34.17 & 18.18 & 15.91 & 16.88 \\
\hline HHConPred & 42.92 & 44.78 & 41.06 & 48.67 & 44.09 & 39.44 \\
\hline ISSEC & 46.44 & 57.89 & 47.13 & 61.53 & 54.56 & 43.23 \\
\hline
\end{tabular}

In each column, the largest element is highlighted in italic. The datasets and the results for all methods except ISSEC were excerpted from [19]

Table 2 Performance of ISSEC and bbcontacts on BetaSheet 186 dataset

\begin{tabular}{llll}
\hline Method & Precision (\%) & Recall (\%) & F-measure (\%) \\
\hline bbcontacts + PSIPRED & 81.1 & 48.2 & 60.5 \\
ISSEC & 68.7 & 66.6 & 62.1 \\
\hline
\end{tabular}

In each column, the largest element is highlighted in italic. The prediction results of bbcontacts were excerpted from [7]

We tested ISSEC on Mem30 and Mem11 datasets and performed comparison with three popular approaches, namely, TMhhcp [14], MemConP [15] and HHConPred [19]. Here we followed the convention used in HHConPred to define $\alpha-\alpha$ contacts, and comparison criterion from HHConPred [19].

As shown in Table 1, on the Mem30 dataset, TMhhcp and MemConP are able to achieve relatively higher values of precision $(61.85 \%$ and $70.68 \%$, respectively) but at the substantial sacrifice of recall (15.48\% and $24.97 \%$, respectively). This leads to relatively low F-measure for these approaches, thus greatly limiting their applications in membrane proteins. In contrast, HHConPred and ISSEC recalled $44.78 \%$ and $57.89 \%$ true $\alpha-\alpha$ contacts and thus achieved higher F-measure than TMhhcp and MemConP. Furthermore, ISSEC significantly outperformed HHConPred by a large margin (> 6\% in F-measure). On the Mem11 dataset, ISSEC outperformed all the three approaches. For example, ISSEC exceeded HHConPred by $13 \%$ in precision, $10 \%$ in recall, and $3 \%$ in F-measure. These results suggested the advantages of ISSEC over the existing approaches.

\section{Prediction accuracy of $\beta-\beta$ contacts on BetaSheet 186 dataset}

Next, we evaluated ISSEC's prediction accuracy of the $\beta-\beta$ contacts on the BetaSheet186 dataset. Here, we followed the convention used in bbcontacts [7] to define true $\beta-\beta$ contacts of proteins.

We compared ISSEC with bbcontacts and summarized the comparison results in Table 2. The table suggested that bbcontacts achieved a higher precision (81.1\%) than ISSEC (68.7\%) but a lower value of recall (48.2\% vs. 66.6\%). As results, ISSEC outperformed bbcontacts by $1.6 \%$ in terms of F-measure. It is worth pointing out that ISSEC exhibited much higher prediction accuracy of $\beta-\beta$ contacts than that of $\alpha-\alpha$ contacts (Additional file 1: Fig. S3). 
It illustrated the extensiveness of our method that a general model for predicting multiple types of inter-SSE contacts could outperform the tools for single one.

\section{Predicting multiple types of inter-SSE contacts simultaneously}

To investigate whether ISSEC could predict multiple types of inter-SSE contacts simultaneously, we evaluated ISSEC on TEST1000 and PSICOV118 datasets. Since there had no published tool for the prediction of multiple types of inter-SSE contacts, we just showed the curves on this two datasets. The proteins in these datasets have both $\alpha-\alpha$ and $\beta-\beta$ contacts. More specifically, the ratio of $\alpha-\alpha, \beta-\beta$ parallel and $\beta-\beta$ anti-parallel contacts is 2:1:2 in TEST1000, and 3:2:4 in PSICOV118.

As shown in Fig. 3, ISSEC could accurately predict inter-SSE contacts on both PSICOV118 (Precision: 63.24\%, Recall: 61.52\%, F-measure: 62.36\%) and TEST1000 dataset (Precision: 57.22\%, Recall: 55.86\%, F-measure: 56.53\%). The performance on PSICOV118 is marginally better than that on the TEST1000 dataset, which might be rooted in the fact that proteins in PSICOV118 usually have more sequence homologs. In addition, for all of the three types of inter-SSE contacts, the prediction accuracy are considerably close, suggesting that ISSEC could apply on proteins with multiple types of inter-SSE contacts.

\section{Applying ISSEC to improve prediction of inter-residue contacts}

As inter-SSE contacts carry coarse-grain information of structure, it is interesting to examine whether this information could be used to improve prediction of inter-residue contacts. For this aim, we integrated the predicted inter-SSE contacts by ISSEC into the deep residual network model designed for inter-residue contact prediction [5]. The original model was denoted as DeepRN model hereafter.

The original DeepRN model was designed to refine the predicted inter-residue contacts generated using co-evolution technique (e.g., CCMpred [22]). The loss function in the original model is cross entropy summed over all residue pairs. Here we enhanced the

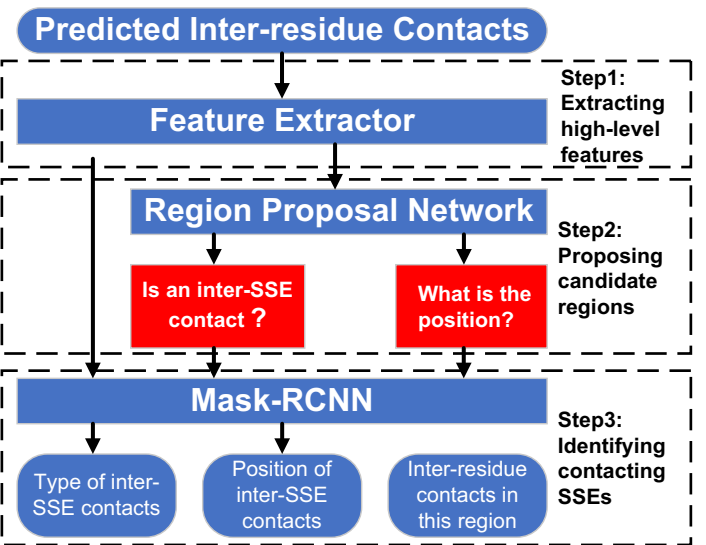

Fig. 3 Deep object detection model used by ISSEC. (i) Feature extractor aims to extract high-level features from the predicted probabilities of inter-residue contacts. (ii) Region proposing network aims to propose candidate rectangle regions that might contain contacting SSEs. (iii) Mask-RCNN aims to judge whether a candidate rectangle region contains characteristic patterns or not 
original DeepRN model with ISSEC as follows: The residue pairs in the predicted contacting SSEs were assigned with higher weight, i.e., for these residue pairs, their loss are multiplied by $1+S_{t}$, where $S_{t}$ represents the score of predicted inter-SSE contact.

$$
\text { Re_weighted Loss }=\sum_{i} \sum_{j}\left(1+S_{t}\right) \times \operatorname{CrossEntropy}(i, j)
$$

Following the widely-used convention, we divided the contacts into short-, mediumand long-range when the sequence distance of the two contacting residues falls into $[6,11],[12,23]$, and $[24,+\infty)$, respectively, and reported the accuracy of top $L / 10, L / 5$, $L / 2$, and $L$ predicted contacts.

As illustrated in Additional file 1: Tables S2 and S3, when enhanced with ISSEC, DeepRN showed considerable performance improvement in most cases. More importantly as shown in Table 3, the performance improvement are more considerable for long-range contacts on both PSICOV118 dataset (4.2\% improvement for top $L$ contacts), CASP11 dataset (3.4\% improvement for top $L$ contacts) and CASP13 dataset (2.0\% improvement for top $L / 10$ contacts). It was well recognized that the prediction of long-range contacts is a challenging task. This result clearly suggested that the global structure information identified by ISSEC could greatly facilitate accurate prediction of inter-residue contacts.

\section{Applying ISSEC to improve 3D structure prediction}

When inter-SSE contacts are known, topology of the full tertiary structures are almost fixed. Here, we used the predicted inter-SSE contacts to guide structure building. Specifically, we integrated ISSEC into CONFOLD [23] as follows: CONFOLD consists of two stages, and at the second stage, it identifies strand-pairs from the structures generated at the first stage and uses these pairs to guide structure building. We replaced the second stage of CONFOLD with ISSEC and compared this hybrid version (denoted as CONFOLD+ISSEC) with the original CONFOLD.

Figure 4 shows the head-to-head comparison of the top model generated by CONFOLD and that by CONFOLD+ISSEC. On 69 out of the 118 proteins in PSICOV118 dataset, CONFOLD+ISSEC generated much better protein structures. We listed 10 of these proteins in Table 4 and exhibited the predicted structures in Fig. 5 and Additional file 1: Figs. S5-S14. Taking protein 101 zA as an example, CONFOLD generated a structure with TMscore of only 0.44, whereas CONFOLD+ISSEC generated a structure with TMscore of 0.55 . For protein $1 \mathrm{i} 4 \mathrm{jA}$ and $1 \mathrm{ctfA}$, the TMscore improvement are even

\begin{tabular}{|c|c|c|c|c|c|c|c|c|c|c|c|c|}
\hline \multirow[t]{2}{*}{ Method } & \multicolumn{4}{|c|}{ Long on PSICOV118 } & \multicolumn{4}{|c|}{ Long on CASP11 } & \multicolumn{4}{|c|}{ Long on CASP13 } \\
\hline & $L / 10$ & $L / 5$ & $L / 2$ & $L$ & $L / 10$ & $L / 5$ & $L / 2$ & $L$ & $L / 10$ & $L / 5$ & $L / 2$ & $L$ \\
\hline CCMpred & 0.714 & 0.591 & 0.384 & 0.242 & 0.472 & 0.416 & 0.326 & 0.244 & 0.326 & 0.302 & 0.218 & 0.153 \\
\hline DeepRN & 0.913 & 0.879 & 0.737 & 0.559 & 0.723 & 0.670 & 0.571 & 0.440 & 0.497 & 0.450 & 0.339 & 0.253 \\
\hline DeepRN+ISSEC & 0.919 & 0.892 & 0.777 & 0.601 & 0.730 & 0.686 & 0.599 & 0.474 & 0.517 & 0.451 & 0.350 & 0.269 \\
\hline
\end{tabular}




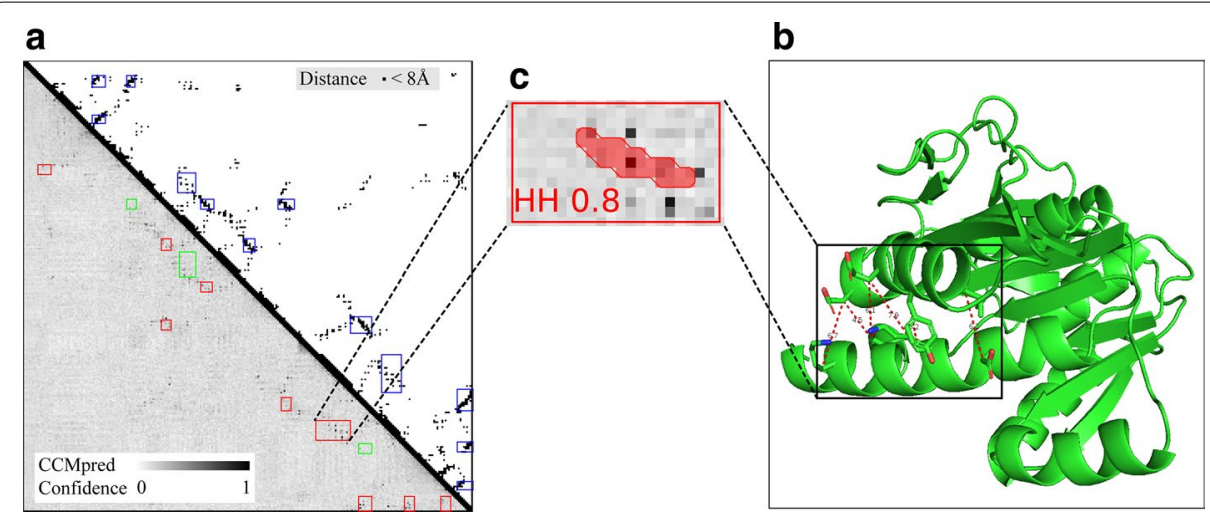

Fig. 4 Prediction of inter-SSE contacts for protein 3 a 4 tA using ISSEC. a The upper-right triangle shows true inter-residue contacts and true contacting SSEs (rectangle regions in blue). The lower-left triangle shows the predicted contacting SSEs and the predicted probability of inter-residue contacts. Here, rectangle regions in red represent true positive prediction where green ones represent incorrect predictions. $\mathbf{b}$ The native 3D-structure of the protein $3 \mathrm{a} 4 \mathrm{tA}$. Here, two contacting helices are shown in the box. $\mathbf{c}$ The prediction results for the contacting helices: type: $\mathrm{HH}$, Confidence score: 0.80 , and masks shown in red

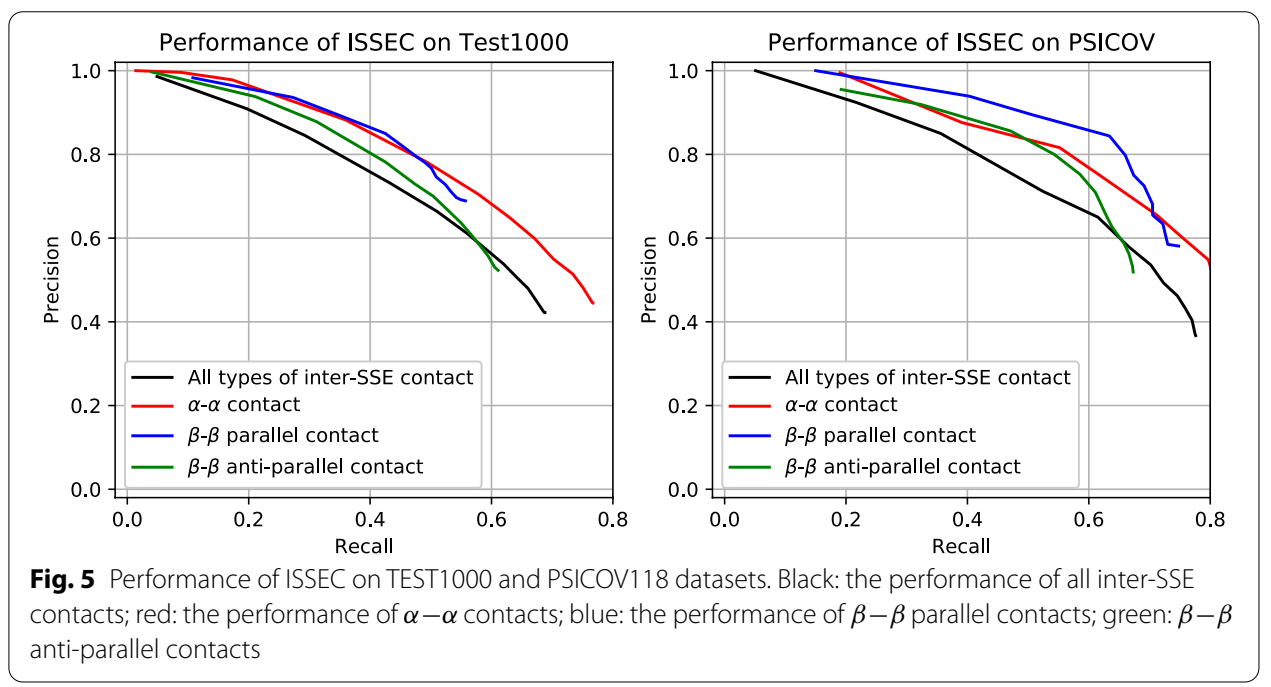

higher than 0.20 . These results suggested that ISSEC could be used to effectively improve tertiary structure prediction.

\section{Discussion}

Our ISSEC could currently predict three frequent types of inter-SSE contacts, i.e., $\alpha-\alpha$, $\beta-\beta$ parallel and $\beta-\beta$ anti-parallel contacts. How to extend ISSEC to predict $\alpha-\beta$ and $\beta$ -turn contacts remains one of the future works.

\section{Conclusions}

In this study, we present an approach to predicting inter-SSE contacts. Experimental results suggested that this approach could be used to predict $\alpha-\alpha$ contacts for membrane proteins and $\beta-\beta$ contacts for $\beta$ proteins. More importantly, it can be used to 
Table 4 Quality of the predicted structures by using CONFOLD and CONFOLD+ISSEC for ten proteins

\begin{tabular}{|c|c|c|c|c|c|}
\hline \multirow[t]{2}{*}{ Target } & \multicolumn{2}{|c|}{ CONFOLD } & \multicolumn{2}{|c|}{ CONFOLD+ISSEC } & \multirow{2}{*}{$\begin{array}{l}\text { TM-score } \\
\text { Improvement }\end{array}$} \\
\hline & RMSD & TM-score & RMSD & TM-score & \\
\hline $1 i 4 j A$ & 13.10 & 0.27 & 11.04 & 0.48 & 0.21 \\
\hline $1 \operatorname{ctfA}$ & 10.62 & 0.29 & 3.90 & 0.49 & 0.20 \\
\hline $1 \mathrm{bdoA}$ & 5.97 & 0.35 & 4.94 & 0.47 & 0.11 \\
\hline $101 \mathrm{zA}$ & 10.26 & 0.44 & 6.43 & 0.55 & 0.11 \\
\hline 1rw1A & 11.31 & 0.28 & 9.28 & 0.39 & 0.11 \\
\hline $1 \mathrm{ktgA}$ & 10.56 & 0.29 & 7.79 & 0.40 & 0.11 \\
\hline $1 \mathrm{pchA}$ & 7.51 & 0.38 & 6.05 & 0.49 & 0.10 \\
\hline 1 tqhA & 4.43 & 0.64 & 3.32 & 0.73 & 0.09 \\
\hline $1 \mathrm{fk} 5 \mathrm{~A}$ & 15.33 & 0.26 & 14.64 & 0.35 & 0.09 \\
\hline InpsA & 13.54 & 0.20 & 11.25 & 0.28 & 0.08 \\
\hline
\end{tabular}

predict multiple types of inter-SSE contacts simultaneously. Furthermore, our approach could be used to improve prediction of both inter-residue contacts and tertiary structure as well.

\section{Methods}

ISSEC infers inter-SSE contacts through detecting rectangle regions with characteristic patterns in the inter-residue contact map. To detect these rectangle regions, ISSEC employs the object detection framework, which was initially proposed for detecting objects in images [24]. For example, given the image shown in Additional file 1: Fig. S2, the goal is to identify both position and type of the objects (two cats, a dog and a duck in this image).

The similarity between inter-residue contact maps and images enables us to apply the object detection technique to infer inter-SSE contacts. Specifically, ISSEC takes the predicted inter-residue contact map as input, and outputs a group of objects representing contacting SSEs (Fig. 6). For each contacting SSE, ISSEC reports its type $(\alpha-\alpha, \beta-\beta$ parallel/anti-parallel contact, further details in Additional file 1), its position (shown as a rectangle), and a confidence score.

However, despite the similarities between inter-residue contact maps and images, the contacting SSEs differ greatly from the objects in images. To fit in the specific properties of inter-SSE contacts, we modified the generic object detection framework. Figure 7 shows the main steps of ISSEC, which are described in more details as follows:

1 Extracting high-level features: To overcome the drawback of directly using the predicted probabilities of inter-residue contact, ISSEC extracts high-level features using a feature pyramid network (FPN, [25]). FPN uses both bottom-up and top-down pathways linked through lateral connections, enabling it to generate a collections of feature maps at multiple levels for subsequent analysis.

2 Proposing candidate rectangle regions: As we have no knowledge of location or size of the rectangle regions formed by contacting SSEs in advance, we propose multiple candidate rectangle regions and expect at least one of these proposed regions to 


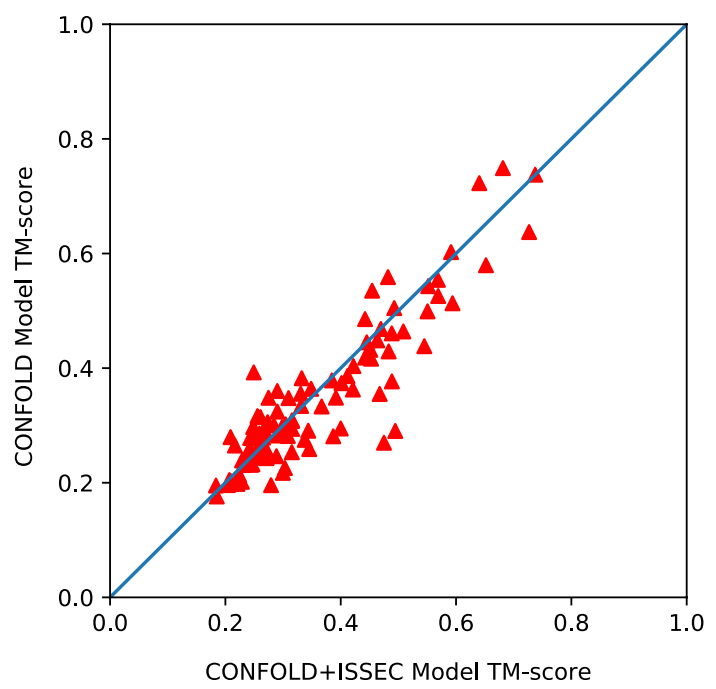

Fig. 6 Head-to-head comparison of the structure quality predicted by CONFOLD and CONFOLD+ISSEC. Dataset: PSICOV118
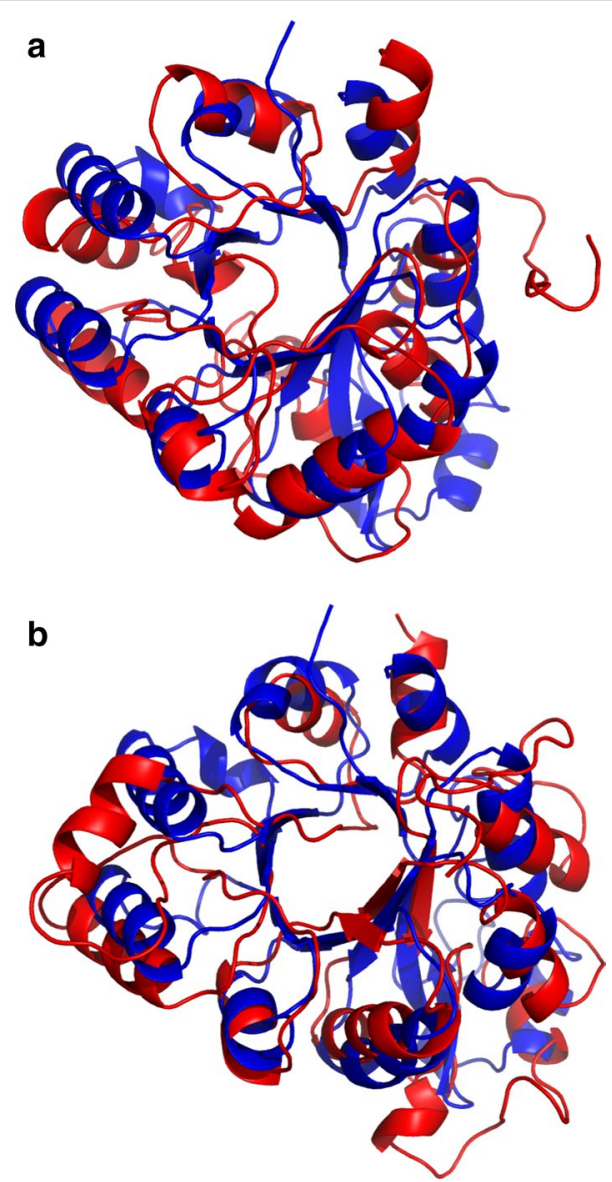

Fig. 7 Predicted structure for protein $101 \mathrm{zA}$ by using CONFOLD (a) and CONFOLD+ISSEC (b). Here the predicted structures are shown in red whereas the native structure is shown in blue 
cover the contacting SSEs. ISSEC accomplishes this objective using a region proposal network (RPN, [26]), which operates in two steps: (1) Generating seed rectangle region on feature maps: ISSEC scans the generated feature maps and generates 9 rectangle regions circled at each element on feature maps. (2) Tracing back to the input interresidue contact map: From each rectangle region on the feature map, ISSEC traces back to a larger rectangle region on the input inter-residue contact map. This tracing back operation is accomplished using ROIAlign [27].

It is worthy pointing out that the rectangle regions formed by contacting SSEs are relatively small; more specifically, each of these regions does not exceed $1 / 4$ of the input inter-residue contact map. This observation provides the possibility to cover all contacting SSEs using the proposed candidate rectangle regions. ISSEC achieves this objective through appropriately choosing various shape and size of the rectangle regions on the feature maps (see Additional file 1: Table S1 for details).

3 Identifying contacting SSEs: For each of the proposed candidate rectangle regions, ISSEC calculates a confidence score to measure whether this region contains characteristic patterns of inter-SSEs or not. Based on the characteristic pattern, ISSEC also determines the type of inter-SSEs contacts, i.e., $\alpha-\alpha, \beta-\beta$ parallel or anti-parallel. In addition, since the candidate rectangle region might be larger than the true size of contacting SSEs, a calibrating operation is also needed to shrink the rectangle region and calculate the true position of the contacting SSEs as well. ISSEC employs the Mask-RCNN [27] technique to achieve these objectives.

Unlike the task of detecting objects in images, the output of Mask-RCNN cannot be directly used to infer contacting SSEs. The reasons are rooted in the difference between the objects in images and contacting SSEs: (1) as shown in Additional file 1: Fig. S2, two objects in images, say the cat and the dog, overlap significantly. In contrast, two rectangle regions formed by contacting SSEs never overlap. (2) Moreover, a rectangle region formed by contacting SSEs would not overlap with diagonal line.

To identify non-overlapping rectangle regions, ISSEC uses a greedy selection strategy that works as follows: We first filter out the rectangle regions that overlap with diagonal and the regions whose confidence score less than a threshold $(T=0.70$ in this study). Next, we sort the remainder in the decreasing order of their confidence score. Then, we select the top rectangle region of the remainder and remove any rectangle regions that overlap with the selected one. This selecting and removing step is repeated until all candidate rectangle regions were processed.

For each of the selected non-overlapping rectangle regions, ISSEC reports the corresponding inter-SSE contact type, position, and confidence score as final results. Finally, a rectangle region that had $>80 \%$ overlap with a native contact was correct one.

\section{Loss function design}

In the training process, ISSEC uses the multi-task loss, including classification loss, localization loss and mask loss (further details in Additional file 1).Briefly speaking, the classification loss measures the difference between the predicted and true types of contacting SSEs. The localization loss measures the difference between the position of rectangle region and the true position of SSEs. The localization loss enables 
ISSEC to acquire accurate position of SSEs, thus avoiding the needs of pre-defined SSE boundaries.

Unlike the $\beta-\beta$ parallel and anti-parallel contacts, the $\alpha-\alpha$ contacts appear as dashed lines, which is unsuitable for the mask mechanism. In this study, we set the weight of mask loss smaller than those of the classification and localization losses.

\section{Supplementary information}

Supplementary information accompanies this paper at https://doi.org/10.1186/s12859-020-03793-y.

Additional file 1: Supplementary methods, tables and figures.

\section{Abbreviations}

ISSEC: Inferring secondary structure element contact; SSE: Secondary structure element; NMR: Nuclear magnetic resonance; HMM: Hidden Markov model; RPN: Region proposal network.

\section{Acknowledgements}

We greatly appreciate Haipeng Gong and Dapeng Xiong from Tsinghua University for providing data.

\section{Authors' contributions}

DB conceived the study, and QZ, DB and JW designed the work. QZ implemented the ISSEC approach. QZ, JW, FS, LP, and SW analyzed the results. QZ and DB wrote the manuscript. All authors read and approved the final manuscript.

\section{Funding}

This work was supported by the National Key Research and Development Program of China (2018YFC0910405), and the National Natural Science Foundation of China (31671369, 31770775). The National Key Research and Development Program of China (2018YFC0910405) covered the design of the study as well as the collection, analysis and interpretation of data, the National Natural Science Foundation of China $(31671369,31770775)$ provided the funding for the experiments and the manuscript writing.

Availability of data and materials

The datasets generated and/or analysed during the current study, together with the codes are available via https://githu b.com/bigict/ISSEC/.

Ethics approval and consent to participate

Not applicable.

\section{Consent for publication}

Not applicable.

Competing interests

The authors declare that they have no competing interests.

\section{Author details}

${ }^{1}$ Key Lab of Intelligent Information Processing, Big Data Academy, Institute of Computing Technology, Chinese Academy of Sciences, Beijing 100190, China. ${ }^{2}$ School of Computer Science, University of Chinese Academy of Sciences, Beijing, China. ${ }^{3}$ Institute of Theoretical Physics, Chinese Academy of Sciences, Beijing 100190, China.

Received: 11 July 2020 Accepted: 30 September 2020

Published online: 05 November 2020

\section{References}

1. Branden Cl, et al. Introduction to protein structure. New York: Garland Science; 1999.

2. Floudas CA. Computational methods in protein structure prediction. Biotechnol Bioeng. 2007;97(2):207-13.

3. Kim DE, DiMaio F, Yu-Ruei Wang R, Song Y, Baker D. One contact for every twelve residues allows robust and accurate topology-level protein structure modeling. Proteins Struct Funct Bioinform. 2014;82:208-18.

4. Zhu J, Zhang H, Li SC, Wang C, Kong L, Sun S, Zheng W-M, Bu D. Improving protein fold recognition by extracting fold-specific features from predicted residue-residue contacts. Bioinformatics. 2017:33(23):3749-57.

5. Wang S, Sun S, Li Z, Zhang R, Xu J. Accurate de novo prediction of protein contact map by ultra-deep learning model. PLoS Comput Biol. 2017;13(1):1-34. https://doi.org/10.1371/journal.pcbi.1005324.

6. Skolnick J, Kolinski A, Ortiz AR. MONSSTER: a method for folding globular proteins with a small number of distance restraints 1. J Mol Biol. 1997;265(2):217-41.

7. Andreani J, Söding J. Bbcontacts: prediction of $\beta$-strand pairing from direct coupling patterns. Bioinformatics. 2015;31(11):1729-37.

8. Barth P, Schonbrun J, Baker D. Toward high-resolution prediction and design of transmembrane helical protein structures. Proc Natl Acad Sci. 2007;104(40):15682-7. 
9. Eilers M, Patel AB, Liu W, Smith SO. Comparison of helix interactions in membrane and soluble $\alpha$-bundle proteins. Biophys J. 2002;82(5):2720-36.

10. Ruczinski I, Kooperberg C, Bonneau R, Baker D. Distributions of beta sheets in proteins with application to structure prediction. Proteins Struct Funct Bioinform. 2002;48(1):85-97.

11. Lo A, Chiu Y-Y, Rødland EA, Lyu P-C, Sung T-Y, Hsu W-L. Predicting helix-helix interactions from residue contacts in membrane proteins. Bioinformatics. 2009;25(8):996-1003.

12. Fuchs A, Kirschner A, Frishman D. Prediction of helix-helix contacts and interacting helices in polytopic membrane proteins using neural networks. Proteins Struct Funct Bioinform. 2009;74(4):857-71.

13. Yang J, Jang R, Zhang Y, Shen H-B. High-accuracy prediction of transmembrane inter-helix contacts and application to GPCR 3D structure modeling. Bioinformatics. 2013;29(20):2579-87.

14. Wang X-F, Chen Z, Wang C, Yan R-X, Zhang Z, Song J. Predicting residue-residue contacts and helix-helix interactions in transmembrane proteins using an integrative feature-based random forest approach. PLoS ONE. 2011;6(10):26767.

15. Hönigschmid P, Frishman D. Accurate prediction of helix interactions and residue contacts in membrane proteins. J Struct Biol. 2016;194(1):112-23.

16. Baldi P, Pollastri G, Andersen CA, Brunak S. Matching protein beta-sheet partners by feedforward and recurrent neural networks. In: Proceedings of the 2000 conference on intelligent systems for molecular biology (ISMB00), La Jolla: AAAI Press; 2000, p. 25-36.

17. Cheng J, Baldi P. Three-stage prediction of protein $\beta$-sheets by neural networks, alignments and graph algorithms. Bioinformatics. 2005;21(suppl-1):75-84

18. Savojardo C, Fariselli P, Martelli PL, Casadio R. BCov: a method for predicting $\beta$-sheet topology using sparse inverse covariance estimation and integer programming. Bioinformatics. 2013;29(24):3151-7.

19. Xiong D, Mao W, Gong H. Predicting the helix-helix interactions from correlated residue mutations. Proteins Struct Funct Bioinform. 2017:85(12):2162-9.

20. Jones DT, Buchan DW, Cozzetto D, Pontil M. PSICOV: precise structural contact prediction using sparse inverse covariance estimation on large multiple sequence alignments. Bioinformatics. 2011;28(2):184-90.

21. Hildebrand PW, Lorenzen S, Goede A, Preissner R. Analysis and prediction of helix-helix interactions in membrane channels and transporters. Proteins Struct Funct Bioinform. 2006;64(1):253-62.

22. Seemayer S, Gruber M, Söding J. CCMpred: fast and precise prediction of protein residue-residue contacts from correlated mutations. Bioinformatics. 2014;30(21):3128-30.

23. Adhikari B, Bhattacharya D, Cao R, Cheng J. CONFOLD: residue-residue contact-guided ab initio protein folding. Proteins Struct Funct Bioinform. 2015;83(8):1436-49.

24. Redmon J, Divvala S, Girshick R, Farhadi A. You only look once: unified, real-time object detection. In: Proceedings of the IEEE conference on computer vision and pattern recognition; 2016, pp. 779-88.

25. Lin T-Y Dollá P, Girshick RB, He K, Hariharan B, Belongie SJ. Feature pyramid networks for object detection. In: The IEEE conference on computer vision and pattern recognition (CVPR); 2017.

26. Ren S, He K, Girshick R, Sun J. Faster R-CNN: towards real-time object detection with region proposal networks. In: Advances in neural information processing systems; 2015, pp. 91-9.

27. He K, Gkioxari G, Dollár P, Girshick R. Mask R-CNN. In: 2017 IEEE international conference on computer vision (ICCV). New York: IEEE; 2017, pp. 2980-8.

\section{Publisher's Note}

Springer Nature remains neutral with regard to jurisdictional claims in published maps and institutional affiliations.

Ready to submit your research? Choose BMC and benefit from:

- fast, convenient online submission

- thorough peer review by experienced researchers in your field

- rapid publication on acceptance

- support for research data, including large and complex data types

- gold Open Access which fosters wider collaboration and increased citations

- maximum visibility for your research: over $100 \mathrm{M}$ website views per year

At BMC, research is always in progress.

Learn more biomedcentral.com/submissions 